\title{
Missing Values in Patient Reported Outcomes: Assessment of Disability in Patients with Low Back Pain
}

\section{TO THE EDITOR:}

Patients are playing an ever-greater role in making decisions about their health and treatment. Consequently, the use of patient reported outcomes (PRO) has increased tremendously in measuring conditions like pain, disability, and quality of life. PRO usage is usually the only option for the assessment of effectiveness of pharmacotherapy in low back pain (LBP). The pain outcomes are analyzed using carefully validated patient reported outcome measurements (PROM) like numeric pain rating scales, Oswestry disability index (ODI), Quebec back pain disability scale (QUEBEC), Roland-Morris disability questionnaire (RMDQ), SF-36, etc.

We have encountered certain pertinent practical issues while assessing these outcomes during our research in patients with LBP. Firstly, we would like to discuss issues in assessing functional disability. Two most commonly used disability scales in patients with LBP are the ODI and QUEBEC. The major issue with this selfreported and interviewed data for these scales is the problem of missing values for the fields involving questions on lifting and running.

The original ODI developed by Fairbank et al (1) has a question regarding lifting, where the patient has to respond according to the level of inconvenience caused due to pain in lifting heavy weights. The original QUEBEC developed by Fritz et al (2) has questions regarding lifting like "Pull or push heavy doors," "Carry two bags of groceries," and "Lift and carry a heavy suitcase." Where the patient has to respond on a 0 to 5 scale where 0 represents "No difficulty at all" and 5 represents "Unable to do."

In both of these cases, while responding to these questions, the patients usually leave these blank. On enquiring about the missing values in the questionnaire from the patients, many answered that they fear that lifting heavy weights may worsen their pain and so, they do not lift any weights. Our understanding was further supported by our observation that, in spite of improvement in pain score, the patients avoid lifting heavy weights and leave the response for the question/s regarding lifting blank.

The validity of these scales lies only in the absence of any missing information, because these scales were developed and validated using every question. Every data item in the questionnaire plays an important role in assessing the outcome. Cutoff level for various disability levels and minimum detectable change are usually calculated using the original author's developed scale summating responses from all questions. Missing data for one to 3 questions may change entire questionnaires' reliability and usefulness.

Another important issue is that despite cross cultural adaptation of these scales, some of the questions may still not be applicable to certain patient groups like women especially older than 45 years in societies of developing nations, where they are commonly not in the habit of running. When female patients were interviewed or self-reported, we observed missing values for the question "Run one block." The reason mentioned was that they are not involved in any running activity.

Thus, it must be emphasized that before adoptions of PROMs for local populations, we must evaluate and revise the instrument through cognitive interviewing, as well as documenting the methods and results to demonstrate that the instructions and item content are appropriate, comprehensive, and understandable to the target population.

Gudala Kapil, PhD Scholar

Department of Pharmacy Practice

National Institute of Pharmaceutical

Education and Research,

SAS Nagar (Mohali), India-160062

Dipika Bansal, MD

Assistant Professor

Department of Pharmacy Practice

National Institute of Pharmaceutical

Education and Research

S.A.S. Nagar (Mohali)-160062 
Punjab, India

E-mail: dipikabansal079@gmail.com

Babita Ghai, MD

Additional Professor

Department of Anaesthesia
Postgraduate Institute of Medical Education and Research

Chandigarh, India

\section{References}

1. Fairbank JC, Pynsent PB. The Oswestry Disability Index. Spine (Phila Pa 1976) 2000; 25:2940-2952.

2. Fritz JM, Irrgang JJ. A comparison of a

modified Oswestry Low Back Pain Disability Questionnaire and the Quebec Back Pain Disability Scale. Phys Ther 2001; 81:776-788. 